\title{
Algorithm for quantum-mechanical finite-nuclear-mass variational calculations of atoms with two $p$ electrons using all-electron explicitly correlated Gaussian basis functions
}

\author{
Keeper L. Sharkey, ${ }^{1}$ Michele Pavanello, ${ }^{1}$ Sergiy Bubin, ${ }^{2,1}$ and Ludwik Adamowicz ${ }^{1,3}$ \\ ${ }^{1}$ Department of Chemistry, University of Arizona, Tucson, Arizona 85721, USA \\ ${ }^{2}$ Quantum Chemistry Research Institute, Kyodai Katsura Venture Plaza 106, Goryo Oohara 1-36, Nishikyo-ku, Kyoto 615-8245, Japan \\ ${ }^{3}$ Department of Physics, University of Arizona, Tucson, Arizona 85721, USA
}

(Received 31 July 2009; published 9 December 2009)

\begin{abstract}
A new algorithm for calculating the Hamiltonian matrix elements with all-electron explicitly correlated Gaussian functions for quantum-mechanical calculations of atoms with two $p$ electrons or a single $d$ electron have been derived and implemented. The Hamiltonian used in the approach was obtained by rigorously separating the center-of-mass motion and it explicitly depends on the finite mass of the nucleus. The approach was employed to perform test calculations on the isotopes of the carbon atom in their ground electronic states and to determine the finite-nuclear-mass corrections for these states.
\end{abstract}

DOI: 10.1103/PhysRevA.80.062510 PACS number(s): 31.15.xt, 31.30.Gs, 31.15.ep, 31.15.V-

\section{INTRODUCTION}

In recent years there has been a renewed interest in very accurate quantum-mechanical calculations of the electronic structures of small atoms [1-7]. The interest has been motivated by both new, more accurate measurements performed on the spectra of these systems and by the development of new computational methods that allow these systems to be described with much higher accuracy than before. Most of the work in the field has been centered on two- and threeelectron atomic systems, which can be very accurately calculated using Hylleraas-type functions. However, the extension of the Hylleraas function approach to atoms with more than three electrons has not been achieved due to complications appearing in determining the Hamiltonian matrix elements [6]. An alternative approach to perform very accurate calculations on atoms, which is not restricted to systems with two or three electrons, has been based on employing explicitly all-electron correlated Gaussian functions. For several years now, this research group has been actively contributing to the development of the Gaussian function approach for very accurate atomic calculations [8-13]. The key features of this approach have been the explicit inclusion of the nuclear motion in the nonrelativistic Hamiltonian and the use of the analytical energy gradient determined with respect to the Gaussian exponential parameters in the variational optimization of the wave functions expanded in terms of the Gaussians. In a recent work on the beryllium atom [13] we demonstrated that calculations utilizing large basis sets of Gaussians that include lowest-order relativistic and quantum electrodynamics (QED) corrections can reproduce the lowest excitation transition energy of this system with the accuracy matching that of the most accurate experiments.

In order to extend correlated Gaussian calculations to larger systems and to a wider range of electronic states, procedures have to be developed to deal with electronic configurations that include electrons with nonzero angular momentum. The first step in this direction was the development and implementation of algorithms for calculating energy and energy gradient for atomic states with one $p$ electron [10]. These algorithms were recently employed [11] in very accu- rate calculations of some $S \rightarrow P$ transition energies of the Be atom. Some other applications to four- and five-electron atomic systems are forthcoming. The next step in extending the capability of the approach utilizing Gaussians in atomic calculations is the development of algorithms for calculating Hamiltonian matrix elements for systems with either two $p$ electrons or a single $d$ electron. Such development has recently been completed in our laboratory and is described in this work. Derivations of the Hamiltonian matrix elements were carried out using matrix calculus.

The derived algorithms have been implemented and some test calculations concerning the smallest atom with two $p$ electrons in the ground state-the carbon atom-have been performed. The results of these test calculations are shown in Sec. VI. The prototype computer code, which has been written, is not yet in a form that can be used to perform largescale calculations involving thousands of Gaussian basis functions. To run such calculations an algorithm for calculating the analytical energy gradient has to be implemented. This task is currently being pursued. Procedures for calculating the lowest-order relativistic corrections will also need to be implemented. Only then can calculations of transition energies for the carbon atom produce results with an adequate accuracy. Even though very precise calculations of the electronic states of the carbon atom is our ultimate goal in this work, as this atom plays a central role in all biomolecules, as well as in many molecular systems found in the interstellar space [14-18], we are also planning to perform calculations of excited electronic states of the beryllium and boron atoms, as well as singly and multiply charged ions of the nitrogen and oxygen atoms. Hence, the present development opens to us a new array of systems and states where the high accuracy quantum calculations can be tested against most accurate high-resolution spectroscopic experiments.

\section{HAMILTONIAN}

Consider a nonrelativistic atomic system consisting of $N$ total particles; $N-1$ total electrons and a nucleus. The $i$ th particle has mass $M_{i}$, charge $Q_{i}$, and a position vector in the 
laboratory Cartesian coordinate frame $\mathbf{R}_{i}$. The nonrelativistic Hamiltonian for the system is

$$
\hat{H}_{\mathrm{lab}}=-\sum_{i=1}^{N} \frac{1}{2 M_{i}} \nabla_{\mathbf{R}_{i}}^{2}+\sum_{i>j=1}^{N} \frac{Q_{i} Q_{j}}{R_{i j}},
$$

where $\boldsymbol{\nabla}_{\mathbf{R}_{i}}$ is the gradient with respect to $\mathbf{R}_{i}$, and $R_{i j}$ is the distance between the $i$ th and $j$ th particles, $R_{i j}=\left|\mathbf{R}_{j}-\mathbf{R}_{i}\right|$.

The center-of-mass motion of the system can be separated out of the laboratory-frame Hamiltonian (1) to yield a Hamiltonian describing the center-of-mass motion and another describing the internal motion of the electrons relative to the nucleus. This is accomplished by placing the nucleus at the origin of a new internal Cartesian coordinate system such that it becomes the reference particle for all other particles in the system (electrons in the case of an atom). The internal coordinates are defined as: $\mathbf{r}_{i}=\mathbf{R}_{i+1}-\mathbf{R}_{1}$. With the three coordinates describing the center of mass denoted as $\mathbf{r}_{0}$ and the total mass of the system being $M_{\mathrm{tot}}=\sum_{i=1}^{N} M_{i}$, the coordinate transformation is

$$
\begin{gathered}
\mathbf{r}_{0}=\frac{M_{1}}{M_{\mathrm{tot}}} \mathbf{R}_{i}+\frac{M_{2}}{M_{\mathrm{tot}}} \mathbf{R}_{2}+\cdots+\frac{M_{N}}{M_{\mathrm{tot}}} \mathbf{R}_{N}, \\
\mathbf{r}_{1}=-\mathbf{R}_{1}+\mathbf{R}_{2}, \\
\mathbf{r}_{2}=-\mathbf{R}_{1}+\mathbf{R}_{3}, \\
\vdots \\
\mathbf{r}_{n}=-\mathbf{R}_{1}+\mathbf{R}_{N} .
\end{gathered}
$$

The internal Hamiltonian obtained using the above coordinate transformation depends only on the internal coordinates, and for an atom, as one can describe, represents the motion of the $n=N-1$ pseudoelectrons in the central field of the nucleus. Denoting the mass of the nucleus as $m_{0}$ and the charge as $q_{0}$, the internal Hamiltonian is

$$
\begin{aligned}
\hat{H}= & -\frac{1}{2}\left(\sum_{i=1}^{n} \frac{1}{\mu_{i}} \nabla_{\mathbf{r}_{i}}^{2}+\sum_{i=1, j=1, i \neq j}^{n} \frac{1}{m_{0}} \nabla_{\mathbf{r}_{i}}^{\prime} \nabla_{\mathbf{r}_{j}}\right)+\sum_{i=1}^{n} \frac{q_{0} q_{i}}{r_{i}} \\
& +\sum_{i>j=1}^{n} \frac{q_{i} q_{j}}{r_{i j}},
\end{aligned}
$$

with the pseudoelectron charges denoted as $q_{i}=Q_{i+1}$ and their reduced masses being $\mu_{i}=m_{0} m_{i} /\left(m_{0}+m_{i}\right)$, where $m_{i}=M_{i+1}$. The particles described by the Hamiltonian (3) are called pseudoelectrons because, even though they have the same charges as the electrons, their masses are reduced electron masses. The motions of the pseudoelectrons are coupled through the mass polarization term, $-\frac{1}{2} \sum_{i=1, j=1, i \neq j}^{n}$ $\left(1 / m_{0}\right) \boldsymbol{\nabla}_{\mathbf{r}_{i}}^{\prime} \nabla_{\mathbf{r}_{i}}$, and through the Coulombic interactions. The prime in the mass polarization term indicates the matrix or vector transpose; this notation is used throughout this work. The Coulombic interactions are dependent on the distances between the pseudoparticles and the origin of the internal coordinate system, $r_{i}$, and on the relative distances between the pseudoparticles, $r_{i j}$, where $r_{i j}=\left|\mathbf{r}_{j}-\mathbf{r}_{i}\right|$.

\section{BASIS FUNCTIONS}

The basis function suitable for describing an $n$-pseudoelectron system with two $p$ electrons (or, more generally, two electrons with arbitrary orbital quantum numbers) can be obtained with a standard procedure of adding angular momenta. Suppose that orbital quantum numbers of electrons $i$ and $j$ are $l_{i}$ and $l_{j}$ (all other electrons are assumed to be in the $s$ state). Using the bra-ket notation the "angular" part of the wave function of the system corresponding to the total orbital angular-momentum number $L$ and the projection on the $z$ axis, $M$ can be represented as the following linear combination:

$$
|L M\rangle=\sum_{\substack{m_{i}, m_{j} \\ m_{i}+m_{j}=M}}\left(L M \mid l_{i} m_{i} l_{j} m_{j}\right)\left|l_{i} m_{i}\right\rangle\left|l_{j} m_{j}\right\rangle,
$$

where the $\left(L M \mid l_{i} m_{i} l_{j} m_{j}\right)$ factors are the Clebsch-Gordan coefficients (see, for example [19], ). The radial (i.e., rotationally invariant) part of the wave function in our case will be in the form of Gaussians. Using the explicit form of spherical harmonics in Cartesian coordinates and tables of the ClebshGordan coefficients one can easily show that for a state of an atom with $L=1$ (and, for certainty, $M=0$ ) containing two $p$ electrons, such as the ground state of the carbon atom, formula (4) becomes

$$
x_{i} y_{j}-x_{j} y_{i},
$$

where the common constant factor and the $1 /\left(r_{i}^{l_{i}} r_{j}^{l_{j}}\right)$ factor were dropped. Thus, suitable explicitly correlated Gaussian basis functions for the calculations of the ground state of the carbon atom are

$$
\phi_{k}=\left(x_{i_{k}} y_{j_{k}}-x_{j_{k}} y_{i_{k}}\right) \exp \left[-\mathbf{r}^{\prime}\left(A_{k} \otimes I_{3}\right) \mathbf{r}\right]
$$

where $i_{k}$ and $j_{k}$ are fixed integers that indicate the $p$ electrons, $A_{k}$ is an $n \times n$ symmetric matrix, the subscript $k$ reflects the fact that the matrix elements are unique for each basis function, $\otimes$ is the Kronecker product, $I_{3}$ is a $3 \times 3$ identity matrix, and $\mathbf{r}$ is a $3 n$ vector that has the form

$$
\mathbf{r}=\left(\begin{array}{c}
\mathbf{r}_{1} \\
\mathbf{r}_{2} \\
\vdots \\
\mathbf{r}_{n}
\end{array}\right)=\left(\begin{array}{c}
x_{1} \\
y_{1} \\
z_{1} \\
\vdots \\
x_{n} \\
y_{n} \\
z_{n}
\end{array}\right) \text {. }
$$

For the sake of simplicity, we will write basis functions as

$$
\phi_{k}=\left(x_{i_{k}} y_{j_{k}}-x_{j_{k}} y_{i_{k}}\right) \exp \left[-\mathbf{r}^{\prime} \mathbf{A}_{k} \mathbf{r}\right] \text {, }
$$

where $\mathbf{A}_{k}=A_{k} \otimes I_{3}$.

Using a general quadratic form in place of $\left(x_{i_{k}} y_{j_{k}}-x_{j_{k}} y_{i_{k}}\right)$ allows for a more generalized approach in deriving the matrix elements. We define our basis functions as 


$$
\phi_{k}=\left(\mathbf{r}^{\prime} \mathbf{W}_{k} \mathbf{r}\right) \exp \left[-\mathbf{r}^{\prime} \mathbf{A}_{k} \mathbf{r}\right],
$$

where $\mathbf{W}_{k}$ is a sparse $3 n \times 3 n$ symmetric matrix comprising only four nonzero elements, two of which have values of $1 / 2$ the other two $-1 / 2$. The $1 / 2$ elements are placed in the $\left(3 i_{k}\right.$ $\left.-2,3 j_{k}-1\right)$ and $\left(3 j_{k}-1,3 i_{k}-2\right)$ positions, while the $-1 / 2$ elements are placed in $\left(3 j_{k}-2,3 i_{k}-1\right)$ and $\left(3 i_{k}-1,3 j_{k}-2\right)$ positions. It should be noted that, in general, we could use a nonsymmetric matrix $\mathbf{W}_{k}$ with only two nonzero elements (yielding the same quadratic form) since there are only two terms in Eq. (8). However, in practice it is much more convenient to deal with symmetric matrices as the derivations of matrix elements becomes considerably simpler in this case.

Furthermore, $\mathbf{W}_{k}$ matrix can be easily changed to obtain basis functions suitable for calculations of systems in $D$ states (i.e., $L=2)$. In the case of a $D$ state formed by a single $d$ electron $\left(l_{i}=2, l_{j}=0, L=2, M=0\right)$, the basis functions are

$$
\phi_{k}=\left(x_{i_{k}}^{2}+y_{i_{k}}^{2}-2 z_{i_{k}}^{2}\right) \exp \left[-\mathbf{r}^{\prime} \mathbf{A}_{k} \mathbf{r}\right] .
$$

If a $D$ state is formed by two $p$ electrons $\left(l_{i}=1, l_{j}=1, L\right.$ $=2, M=0)$, the basis functions should be

$$
\phi_{k}=\left(x_{i_{k}} x_{j_{k}}+y_{i_{k}} y_{j_{k}}-2 z_{i_{k}} z_{j_{k}}\right) \exp \left[-\mathbf{r}^{\prime} \mathbf{A}_{k} \mathbf{r}\right] .
$$

Finally, the most trivial case of an $S(L=0, M=0)$ state of an atom formed by two $p$ electrons should be described with spherically symmetric basis functions,

$$
\begin{aligned}
\phi_{k} & =\left(x_{i_{k}} x_{j_{k}}+y_{i_{k}} y_{j_{k}}+z_{i_{k}} z_{j_{k}}\right) \exp \left[-\mathbf{r}^{\prime} \mathbf{A}_{k} \mathbf{r}\right] \\
& =\mathbf{r}_{i_{k}}^{\prime} \mathbf{r}_{j_{k}} \exp \left[-\mathbf{r}^{\prime} \mathbf{A}_{k} \mathbf{r}\right] .
\end{aligned}
$$

The basis functions used in a bound-state calculation must be square integrable which effectively imposes restrictions on the $A_{k}$ matrix. The $A_{k}$ matrix must be positive definite. Rather than restricting the $A_{k}$ matrix elements, $A_{k}$ is represented in a Cholesky factored form as $A_{k}=L_{k} L_{k}^{\prime}$, where $L_{k}$ is a lower triangular matrix. With this representation, $A_{k}$ is automatically positive definite. It should also be mentioned that this form of $A_{k}$ matrix does not limit the flexibility of basis functions since any allowable choice of $A_{k}$ matrix can be represented by some $L_{k}$ matrix, because any symmetric positive matrix can be represented in a Cholesky factored form.

\section{PERMUTATIONAL SYMMETRY}

The trial wave function and the basis functions used in the wave-function expansion should possess the proper symmetry with respect to the permutations of identical particles involved in the system. This symmetry depends on whether the particles are bosons or fermions. Since the Hamiltonian is spin independent, the spin can be completely eliminated from the symmetry consideration, leading to a nontrivial symmetry projection operator, $\hat{Y}$, that needs to be applied to the spatial wave function and, thus, to each basis function. This operator is a linear combination of some permutation operators. In calculating the overlap and Hamiltonian matrix elements all the permutations can be applied to the ket as $\hat{P}=\hat{Y}^{\dagger} \hat{Y}$ (the dagger stands for conjugate). This results in cer- tain sums of elementary matrix elements in which the ket is affected by some permutation,

$$
\left\langle\phi_{k}|\hat{P}| \phi_{l}\right\rangle=\left\langle\phi_{k} \mid \widetilde{\phi}_{l}\right\rangle \text { and }\left\langle\phi_{k}|\hat{H} \hat{P}| \phi_{l}\right\rangle=\left\langle\phi_{k}|\hat{H}| \widetilde{\phi}_{l}\right\rangle \text {. }
$$

Here $\widetilde{\phi}_{l}$ stands for permuted $\phi_{l}$. The calculation of matrix elements with permuted kets can be done using the same procedure as for nonpermuted ones and, therefore, it is required to only transform elements of matrices $\mathbf{A}_{l}$ and $\mathbf{W}_{l}$ accordingly, i.e.,

$$
\begin{aligned}
& \hat{P}\left(\mathbf{r}^{\prime} \mathbf{W}_{l} \mathbf{r}\right) \exp \left[-\mathbf{r}^{\prime} \mathbf{A}_{l} \mathbf{r}\right] \\
& \quad=\left(\mathbf{r}^{\prime} \mathbf{P}^{\prime} \mathbf{W}_{l} \mathbf{P r}\right) \exp \left[-\mathbf{r}^{\prime} \mathbf{P}^{\prime} \mathbf{A}_{l} \mathbf{P r}\right],
\end{aligned}
$$

where $\mathbf{P}=P \otimes I_{3}$ is the permutation matrix corresponding to permutation operator $\hat{P}$.

To build the permutation symmetry projector for fermions, a Young tableaux is constructed. For $n$ fermions (electrons) and for the total spin of the system being $s$, the symmetry quantum number, $p$, is calculated as $p=\frac{n}{2}-s$. Then a partition is defined as $\mu=\left[2^{p} 1^{n-2 p}\right]$. The partition describes the structure of the Young tableaux for the state of the system under consideration. The tableaux consists of two boxes in the first $p$ rows and one box in the remaining $n-2 p$ rows. The carbon atom with six electrons and the total spin in the ground state of one has a partition of $\mu=\left[2^{2} 1^{2}\right]$ and the following Young tableaux:

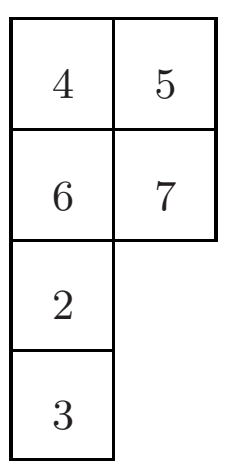

where the following particle numbering convention is used: the nucleus is labeled as " 1 ," electrons " 2 " and " 3 " represent the $p$ electrons, and electrons " 4 ," "5," “6," and "7" are spin-paired $s$ electrons. The permutation symmetry projector can now be constructed using the Young tableaux in the following way:

$$
\hat{Y}=\hat{S} \hat{A}
$$

where $\hat{S}$ and $\hat{A}$ are idempotent symmetrization and antisymmetrization operators. $\hat{A}$ is constructed as a product of antisymmetrizers over particles in each column of the Young tableaux. $\hat{S}$ is constructed as a product of symmetrizers over particles in each row. Thus, in the present case $\hat{A}=\left(\hat{1}-\hat{P}_{46}\right)\left(\hat{1}-\hat{P}_{26}-\hat{P}_{24}\right)\left(\hat{1}-\hat{P}_{32}-\hat{P}_{36}-\hat{P}_{34}\right)\left(\hat{1}-\hat{P}_{57}\right) . \hat{1}$ is the identity operator. The symmetrization operator is: $\hat{S}$ 
$=\left(\hat{1}+\hat{P}_{45}\right)\left(\hat{1}+\hat{P}_{67}\right)$. As mentioned above, the operator which should be applied to $\phi_{l}$ is $Y^{\dagger} Y$.

\section{MATRIX ELEMENTS}

In the beginning, it is necessary to briefly explain the notation scheme used throughout this work. The scheme is the same as the one used in our previous work [10]. As a rule, small Greek letters are used to denote scalar quantities. Bold small Greek letters represent usual three-component vectors. Capital Greek letters are used for $3 \times 3$ matrices. Small Latin letters normally denote $n$-component vectors ( $n$ is the number of pseudoparticles). Bold small Latin letters should be understood as $3 n$-component vectors. If such a letter has an index that means it is a three-component vectors. For example, $\mathbf{r}$ is a $3 n$-component vector, while $\mathbf{r}_{i}$ is a three-component vector. Capital Latin letters are used for $n$ $\times n$ matrices. At last, bold capital Latin letters are $3 n \times 3 n$ matrices. Vertical bars around a matrix denote its determinant, while same bars around a vector or a scalar stand for absolute value or length. All matrix-matrix and matrix-vector products that appear in formulas should be treated using conventional matrix algebra. For example, a product of a $p \times q$ matrix and a $q \times k$ matrices yields a $p \times k$ matrix.

Before evaluating the matrix elements, we also need to give a well-known formula for the following general p-dimensional Gaussian integral:

$$
\int_{-\infty}^{+\infty} \exp \left[-x^{\prime} A x+y^{\prime} x\right] d x=\frac{\pi^{p / 2}}{|A|^{1 / 2}} \exp \left[y^{\prime} A^{-1} y\right]
$$

where $x$ is a $p$-component vector of variables, $A$ is a symmetric $p \times p$ positive-definite matrix, $y$ is a $p$-component constant vector, and $A^{-1}$ is the inverse of $A$. The above integral is frequently used in the derivation of the overlap and Hamiltonian matrix elements in this work. It is convenient to rewrite the basis function (9) as a derivative with respect to some parameter $\alpha_{k}$,

$$
\phi_{k}=-\frac{\partial}{\partial \alpha_{k}} \exp \left[-\mathbf{r}^{\prime}\left(\mathbf{A}_{k}+\alpha_{k} \mathbf{W}_{k}\right) \mathbf{r}\right] \mid \alpha_{k 0}
$$

where the following Gaussian function is called the generator function,

$$
\varphi_{k}=\exp \left[-\mathbf{r}^{\prime}\left(\mathbf{A}_{k}+\alpha \mathbf{W}_{k}\right) \mathbf{r}\right]
$$

Gaussian basis functions in the form given by expression (17) are used in the derivations of the overlap and the Hamiltonian matrix elements.

\section{A. Overlap integral}

The overlap integral between two Gaussian basis functions (17) is

$$
\begin{aligned}
& \left\langle\phi_{k} \mid \phi_{l}\right\rangle=\frac{\partial}{\partial \alpha_{k}} \frac{\partial}{\partial \alpha_{l}}\left\langle\varphi_{k} \mid \varphi_{l}\right\rangle \\
& =\left.\frac{\partial}{\partial \alpha_{k}} \frac{\partial}{\partial \alpha_{l}} \int_{-\infty}^{+\infty} \exp \left[-\mathbf{r}^{\prime}\left(\mathbf{A}_{k l}+\alpha_{k} \mathbf{W}_{k}+\alpha_{l} \mathbf{W}_{l}\right) \mathbf{r}\right] d \mathbf{r}\right|_{\alpha_{k}=\alpha_{l}=0},
\end{aligned}
$$

where $\mathbf{A}_{k l} \equiv \mathbf{A}_{k}+\mathbf{A}_{l}$. Equation (16) can be now directly applied yielding the following derivative:

$$
\left\langle\phi_{k} \mid \phi_{l}\right\rangle=\pi^{3 n / 2} \frac{\partial}{\partial \alpha_{k}} \frac{\partial}{\partial \alpha_{l}}\left|\mathbf{A}_{k l}+\alpha_{k} \mathbf{W}_{k}+\alpha_{l} \mathbf{W}_{l}^{-1 / 2}\right|_{\alpha_{k}=\alpha_{l}=0} .
$$

Here, recall that the differential of the determinant of an arbitrary matrix $X$ and its inverse are

$$
d|X|=|X| \operatorname{tr}\left[X^{-1} d X\right],
$$

and,

$$
d X^{-1}=-X^{-1}(d X) X^{-1} .
$$

In Eq. (21) $\operatorname{tr}[\ldots]$ denotes the trace of a matrix. Differentiating Eq. (20) with respect to $\alpha_{l}$ and $\alpha_{k}$ using Eq. (21) and setting $\alpha_{k}=\alpha_{l}=0$ yields

$$
\begin{aligned}
\left\langle\phi_{k} \mid \phi_{l}\right\rangle= & \frac{1}{2} \pi^{3 n / 2}\left|\mathbf{A}_{k l}\right|^{-1 / 2}\left\{\frac{1}{2} \operatorname{tr}\left[\mathbf{A}_{k l}^{-1} \mathbf{W}_{k}\right] \operatorname{tr}\left[\mathbf{A}_{k l}^{-1} \mathbf{W}_{l}\right]\right. \\
& \left.+\operatorname{tr}\left[\mathbf{A}_{k l}^{-1} \mathbf{W}_{k} \mathbf{A}_{k l}^{-1} \mathbf{W}_{l}\right]\right\} .
\end{aligned}
$$

Formula (23) contains $3 n \times 3 n$ matrices. For computing purposes, it is desirable to reduce the dimensions of the matrices from $3 n \times 3 n$ to $n \times n$ by factoring $I_{3}$ using the definition of the Kronecker product whenever possible. The determinant of $\mathbf{A}_{k l}$, which is a $3 n \times 3 n$ matrix, can be reduced to an expression involving the determinant of the $A_{k l}$ matrix, an $n$ $\times n$ matrix. If $\mathbf{X}$ is an $3 n \times 3 n$ matrix in the form $\mathbf{X}=X \otimes I_{3}$, its determinant can be calculated as

$$
|\mathbf{X}|=\left|X \otimes I_{3}\right|=|X|^{3} .
$$

This is the only simplification that can be made in the overlap formula (23), which results in the final form of the overlap matrix element,

$$
\begin{aligned}
\left\langle\phi_{k} \mid \phi_{l}\right\rangle= & \frac{1}{2} \pi^{3 n / 2}\left|A_{k l}\right|^{-3 / 2}\left\{\frac{1}{2} \operatorname{tr}\left[\mathbf{A}_{k l}^{-1} \mathbf{W}_{k}\right] \operatorname{tr}\left[\mathbf{A}_{k l}^{-1} \mathbf{W}_{l}\right]\right. \\
& \left.+\operatorname{tr}\left[\mathbf{A}_{k l}^{-1} \mathbf{W}_{k} \mathbf{A}_{k l}^{-1} \mathbf{W}_{l}\right]\right\} .
\end{aligned}
$$

It is worth mentioning that $I_{3}$ cannot be factored out of the traces in Eq. (25) by applying the following transformation:

$$
\operatorname{tr}[\mathbf{A C}]=\operatorname{tr}\left[A C \otimes I_{3}\right]=3 \operatorname{tr}[A C],
$$

by using the following matrix product property:

$$
(A \otimes B)(C \otimes D)=(A C \otimes B D),
$$

where $\mathbf{A}$ and $\mathbf{C}$ are arbitrary $3 n \times 3 n$ matrices in the following form $\mathbf{A}=A \otimes I_{3}$, and $B$ and $D$ are equal to $I_{3}$. For ex- 
ample, it is not possible to apply the above transformation in the case of the $\mathbf{W}_{k}$ matrix, which is used to generate the pre-exponential angular factor in the basis function (6), because there is no such $n \times n$ matrix $W_{k}$ that satisfies the condition

$$
\mathbf{W}_{k}=W_{k} \otimes I_{3} .
$$

The sparsity of $\mathbf{W}_{k}$ matrix cannot be explicitly exploited at the level of the formulas, but it has to be handled when the formulas are coded in the computer program. This simplification involves eliminating as many as possible multiplications involving zeros which significantly reduces the calculation time.

Additionally, when $\mathbf{W}_{k}$ is specified to describe basis functions for two $p$ electrons of the form $x_{i_{k}} y_{j_{k}} \exp \left[-\mathbf{r}^{\prime} \mathbf{A}_{k} \mathbf{r}\right]$ and $x_{i_{k}} x_{j_{k}} \exp \left[-\mathbf{r}^{\prime} \mathbf{A}_{k} \mathbf{r}\right]$, as seen in Eqs. (8) and (11), $\operatorname{tr}\left[\mathbf{A}_{k l}^{-1} \mathbf{W}_{k}\right]$ and $\operatorname{tr}\left[\mathbf{A}_{k l}^{-1} \mathbf{W}_{l}\right]$ become zero. This fact significantly reduces the final formula for these cases from Eq. (25) to

$$
\left\langle\phi_{k} \mid \phi_{l}\right\rangle=\frac{1}{2} \pi^{3 n / 2}\left|A_{k l}\right|^{-3 / 2} \operatorname{tr}\left[\mathbf{A}_{k l}^{-1} \mathbf{W}_{k} \mathbf{A}_{k l}^{-1} \mathbf{W}_{l}\right] .
$$

This simplification is used throughout the derivation of Hamiltonian matrix elements. However, when $\mathbf{W}_{k}$ is used to describe basis functions for one $d$ electron of the form $x_{i_{k}}^{2}$ as seen in Eq. (10), the traces mentioned above are nonzero and, therefore, Eq. (25) cannot be reduced any further.

\section{B. Kinetic energy integral}

The kinetic-energy operator in Eq. (3) is expressed as a sum that can be written in the following form:

$$
-\frac{1}{2}\left(\sum_{i=1}^{n} \frac{1}{\mu_{i}} \nabla_{\mathbf{r}_{i}}^{2}+\sum_{i=1, j=1, i \neq j}^{n} \frac{1}{m_{0}} \nabla_{\mathbf{r}_{i}}^{\prime} \nabla_{\mathbf{r}_{j}}\right)=-\boldsymbol{\nabla}_{\mathbf{r}}^{\prime} \mathbf{M} \nabla_{\mathbf{r}}
$$

Here the mass matrix $\mathbf{M}$ is $\mathbf{M}=M \otimes I_{3}$, where in the $M$ matrix the diagonal elements are set to $1 /\left(2 m_{1}\right), 1 /\left(2 m_{2}\right), \ldots, 1 /\left(2 m_{n}\right)$, while the off-diagonal elements are set to $1 /\left(2 m_{0}\right)$. Again, $m_{0}$ is the mass of the nucleus and $m_{1}, \ldots$, and $m_{n}$ are the electron masses. Using Eq. (18) the kinetic-energy integral for the basis functions $\phi_{k}$ and $\phi_{l}$ is

$$
\left\langle\phi_{k}\left|-\nabla_{\mathbf{r}}^{\prime} \mathbf{M} \nabla_{\mathbf{r}}\right| \phi_{l}\right\rangle=\frac{\partial}{\partial \alpha_{k}} \frac{\partial}{\partial \alpha_{l}}\left\langle\nabla_{\mathbf{r}} \varphi_{k}|\mathbf{M}| \nabla_{\mathbf{r}} \varphi_{l}\right\rangle .
$$

The gradient operator acting on the generator function produces

$$
\nabla_{\mathbf{r}} \varphi_{k}=-2\left(\mathbf{A}_{k}+\alpha_{k} \mathbf{W}_{k}\right) \mathbf{r} \varphi_{k}
$$

Plugging this into the kinetic-energy integral gives the following four integrals:

$$
\begin{aligned}
\left\langle\nabla_{\mathbf{r}} \varphi_{k}|\mathbf{M}| \nabla_{\mathbf{r}} \varphi_{l}\right\rangle & \\
= & \left\langle-2\left(\mathbf{A}_{k}+\alpha_{k} \mathbf{W}_{k}\right) \mathbf{r} \varphi_{k}|\mathbf{M}|-2\left(\mathbf{A}_{l}+\alpha_{l} \mathbf{W}_{l}\right) \mathbf{r} \varphi_{l}\right\rangle \\
= & 4\left\{\left\langle\varphi_{k}\left|\mathbf{r}^{\prime} \mathbf{A}_{k} \mathbf{M} \mathbf{A}_{l} \mathbf{r}\right| \varphi_{l}\right\rangle+\left\langle\varphi_{k}\left|\mathbf{r}^{\prime} \mathbf{A}_{k} \mathbf{M} \mathbf{W}_{l} \mathbf{r}\right| \varphi_{l}\right\rangle\right. \\
& \left.+\left\langle\varphi_{k}\left|\mathbf{r}^{\prime} \mathbf{W}_{k} \mathbf{M} \mathbf{A}_{l} \mathbf{r}\right| \varphi_{l}\right\rangle+\left\langle\varphi_{k}\left|\mathbf{r}^{\prime} \mathbf{W}_{k} \mathbf{M} \mathbf{W}_{l} \mathbf{r}\right| \varphi_{l}\right\rangle\right\} .
\end{aligned}
$$

These four integrals can be evaluated using the following auxiliary integral:

$$
\begin{aligned}
\left\langle\varphi_{k}\left|\mathbf{r}^{\prime} \mathbf{B r}\right| \varphi_{l}\right\rangle & \\
= & -\left.\frac{\partial}{\partial \beta}\left\langle\varphi_{k}\left|\exp \left[-\beta \mathbf{r}^{\prime} \mathbf{B r}\right]\right| \varphi_{l}\right\rangle\right|_{\beta=0} \\
& =-\left.\pi^{3 n / 2} \frac{\partial}{\partial \beta}\left|\mathbf{A}_{k l}+\alpha_{k} \mathbf{W}_{k}+\alpha_{l} \mathbf{W}_{l}+\beta \mathbf{B}\right|^{-1 / 2}\right|_{\beta=0},
\end{aligned}
$$

where $\beta$ is a parameter and $\mathbf{B}$ is some symmetric matrix. Notice that matrices in the four integrals above are not necessarily symmetric. However, we can always substitute a nonsymmetric matrix $\mathbf{X}$ in a quadratic form with a symmetric one $\left(\mathbf{X}+\mathbf{X}^{\prime}\right) / 2 \rightarrow \mathbf{X}$ while keeping the quadratic form unchanged.

Next, after some straightforward manipulations, one can show that

$$
\begin{aligned}
\left\langle\phi_{k}\left|\mathbf{r}^{\prime} \mathbf{B} \mathbf{r}\right| \phi_{l}\right\rangle=-\frac{1}{2} \pi^{3 n / 2} \frac{\partial}{\partial \beta}\left|\mathbf{A}_{k l}+\beta \mathbf{B}\right|^{-1 / 2} \\
\times\left(\frac{1}{2} \operatorname{tr}\left[\left(\mathbf{A}_{k l}+\beta \mathbf{B}\right)^{-1} \mathbf{W}_{k}\right] \operatorname{tr}\right. \\
\times\left[\left(\mathbf{A}_{k l}+\beta \mathbf{B}\right)^{-1} \mathbf{W}_{l}\right] \\
\left.\times \operatorname{tr}\left[\left(\mathbf{A}_{k l}+\beta \mathbf{B}\right)^{-1} \mathbf{W}_{k}\left(\mathbf{A}_{k l}+\beta \mathbf{B}\right)^{-1} \mathbf{W}_{l}\right]\right)\left.\right|_{\beta=0} \\
=\frac{1}{2} \frac{\pi^{3 n / 2}}{\left|A_{k l}\right|^{3 / 2}}\left(\frac{1}{4} \eta_{1} \eta_{2} \operatorname{tr}\left[\mathbf{A}_{k l}^{-1} \mathbf{B}\right]+\frac{1}{2} \eta_{1} \operatorname{tr}\left[\mathbf{A}_{k l}^{-1} \mathbf{W}_{l} \mathbf{A}_{k l}^{-1} \mathbf{B}\right]\right. \\
+\frac{1}{2} \eta_{2} \operatorname{tr}\left[\mathbf{A}_{k l}^{-1} \mathbf{W}_{k} \mathbf{A}_{k l}^{-1} \mathbf{B}\right]+\frac{1}{2} \eta_{3} \operatorname{tr}\left[\mathbf{A}_{k l}^{-1} \mathbf{B}\right] \\
\left.+\operatorname{tr}\left[\mathbf{A}_{k l}^{-1} \mathbf{W}_{k} \mathbf{A}_{k l}^{-1} \mathbf{W}_{l} \mathbf{A}_{k l}^{-1} \mathbf{B}\right]+\operatorname{tr}\left[\mathbf{A}_{k l}^{-1} \mathbf{W}_{l} \mathbf{A}_{k l}^{-1} \mathbf{W}_{k} \mathbf{A}_{k l}^{-1} \mathbf{B}\right]\right)
\end{aligned}
$$

where we have defined: $\eta_{1}=\operatorname{tr}\left[\mathbf{A}_{k l}^{-1} \mathbf{W}_{k}\right], \eta_{2}=\operatorname{tr}\left[\mathbf{A}_{k l}^{-1} \mathbf{W}_{l}\right]$, and $\eta_{3}=\operatorname{tr}\left[\mathbf{A}_{k l}^{-1} \mathbf{W}_{k} \mathbf{A}_{k l}^{-1} \mathbf{W}_{l}\right]$.

Now, using Eqs. (31), (33), and (35), and making some simplifications, the final formula for the kinetic-energy matrix elements is

$$
\begin{aligned}
\left\langle\phi_{k}\right|- & \nabla_{\mathbf{r}}^{\prime} \mathbf{M} \boldsymbol{\nabla}_{\mathbf{r}}^{\prime}\left|\phi_{l}\right\rangle \\
= & \pi^{3 n / 2}\left|A_{k l}\right|^{-3 / 2}\left\{\frac{1}{2} \eta_{1} \eta_{2} \operatorname{tr}\left[\mathbf{A}_{k l}^{-1} \mathbf{A}_{k} \mathbf{M} \mathbf{A}_{l}\right]\right. \\
& +\eta_{3} \operatorname{tr}\left[\mathbf{A}_{k l}^{-1} \mathbf{A}_{k} \mathbf{M} \mathbf{A}_{l}\right] \\
& +\eta_{1}\left(\operatorname{tr}\left[\mathbf{A}_{k l}^{-1} \mathbf{W}_{l} \mathbf{A}_{k l}^{-1} \mathbf{A}_{k} \mathbf{M} \mathbf{A}_{l}\right]-\operatorname{tr}\left[\mathbf{A}_{k l}^{-1} \mathbf{A}_{k} \mathbf{M} \mathbf{W}_{l}\right]\right) \\
& +\eta_{2}\left(\operatorname{tr}\left[\mathbf{A}_{k l}^{-1} \mathbf{W}_{k} \mathbf{A}_{k l}^{-1} \mathbf{A}_{k} \mathbf{M} \mathbf{A}_{l}\right]-\operatorname{tr}\left[\mathbf{A}_{k l}^{-1} \mathbf{W}_{k} \mathbf{M} \mathbf{A}_{l}\right]\right) \\
& +2\left(\operatorname{tr}\left[\mathbf{A}_{k l}^{-1} \mathbf{W}_{k} \mathbf{A}_{k l}^{-1} \mathbf{W}_{l} \mathbf{A}_{k l}^{-1} \mathbf{A}_{k} \mathbf{M} \mathbf{A}_{l}\right]+\operatorname{tr}\left[\mathbf{A}_{k l}^{-1} \mathbf{W}_{k} \mathbf{M} \mathbf{W}_{l}\right]\right. \\
& +\operatorname{tr}\left[\mathbf{A}_{k l}^{-1} \mathbf{W}_{k} \mathbf{A}_{k l}^{-1} \mathbf{A}_{k} \mathbf{M} \mathbf{W}_{l}\right]+\operatorname{tr}\left[\mathbf{A}_{k l}^{-1} \mathbf{W}_{l} \mathbf{A}_{k l}^{-1} \mathbf{W}_{k} \mathbf{M} \mathbf{A}_{l}\right] \\
& \left.\left.+\operatorname{tr}\left[\mathbf{A}_{k l}^{-1} \mathbf{W}_{l} \mathbf{A}_{k l}^{-1} \mathbf{W}_{k} \mathbf{A}_{k l}^{-1} \mathbf{A}_{k} \mathbf{M} \mathbf{A}_{l}\right]\right)\right\}
\end{aligned}
$$

Similarly to the overlap formula, the kinetic-energy formula 
can be further reduced for basis functions (8) and (11) to

$$
\begin{aligned}
\left\langle\phi_{k}\right|- & \nabla_{\mathbf{r}}^{\prime} \mathbf{M} \boldsymbol{\nabla}_{\mathbf{r}}^{\prime}\left|\phi_{l}\right\rangle \\
= & \pi^{3 n / 2}\left|A_{k l}\right|^{-3 / 2}\left\{\eta_{3} \operatorname{tr}\left[\mathbf{A}_{k l}^{-1} \mathbf{A}_{k} \mathbf{M} \mathbf{A}_{l}\right]\right. \\
& +2\left(\operatorname{tr}\left[\mathbf{A}_{k l}^{-1} \mathbf{W}_{k} \mathbf{A}_{k l}^{-1} \mathbf{W}_{l} \mathbf{A}_{k l}^{-1} \mathbf{A}_{k} \mathbf{M} \mathbf{A}_{l}\right]\right. \\
& +\operatorname{tr}\left[\mathbf{A}_{k l}^{-1} \mathbf{W}_{k} \mathbf{M} \mathbf{W}_{l}\right]+\operatorname{tr}\left[\mathbf{A}_{k l}^{-1} \mathbf{W}_{k} \mathbf{A}_{k l}^{-1} \mathbf{A}_{k} \mathbf{M} \mathbf{W}_{l}\right] \\
& +\operatorname{tr}\left[\mathbf{A}_{k l}^{-1} \mathbf{W}_{l} \mathbf{A}_{k l}^{-1} \mathbf{W}_{k} \mathbf{M} \mathbf{A}_{l}\right] \\
& \left.\left.+\operatorname{tr}\left[\mathbf{A}_{k l}^{-1} \mathbf{W}_{l} \mathbf{A}_{k l}^{-1} \mathbf{W}_{k} \mathbf{A}_{k l}^{-1} \mathbf{A}_{k} \mathbf{M} \mathbf{A}_{l}\right]\right)\right\} .
\end{aligned}
$$

\section{Dirac delta function and potential energy integral}

The potential-energy operator in the internal Hamiltonian (3) depends on the inverses of the interparticle distances between the electrons and between the electrons and the nuclei, $r_{i}$ and $r_{i j}$, and, therefore, a more general elemental integral can be considered,

$$
\left\langle\phi_{k}\left|r_{i j}^{\nu}\right| \phi_{l}\right\rangle, \quad \nu>-3,
$$

which gives the integrals required for computing the potential-energy matrix elements when $\nu=-1$. To obtain the expression (38) we will first evaluate an auxiliary integral that involves the following three-dimensional Dirac delta function:

$$
\begin{aligned}
& \delta\left(a_{1} \mathbf{r}_{1}+a_{2} \mathbf{r}_{2}+\ldots+a_{n} \mathbf{r}_{n}-\boldsymbol{\xi}\right) \\
& \quad \equiv \delta\left(\left(a \otimes I_{3}\right)^{\prime} \mathbf{r}-\boldsymbol{\xi}\right) \\
& \quad=\lim _{\beta \rightarrow \infty}\left(\frac{\beta}{\pi}\right)^{3 / 2} \exp \left\{-\beta\left[\left(a \otimes I_{3}\right)^{\prime} \mathbf{r}-\xi\right]^{2}\right\},
\end{aligned}
$$

where $a$ is a real $n$-component vector, $\boldsymbol{\xi}$ is a real threedimensional parameter, and $\beta$ is a parameter. When we set $a=e^{i}-e^{j}$, where $e^{m}$ is an $n$-component vector whose $m$ th component is 1 and all others are zeros, then Eq. (38) can be expressed as

$$
\begin{aligned}
\left\langle\phi_{k}\left|r_{i j}^{\nu}\right| \phi_{l}\right\rangle= & \int_{-\infty}^{+\infty}|\boldsymbol{\xi}|^{\nu}\left\langle\phi_{k}\left|\delta\left(\left(a \otimes I_{3}\right)^{\prime} \mathbf{r}-\boldsymbol{\xi}\right)\right| \phi_{l}\right\rangle d \boldsymbol{\xi} \\
= & \int_{-\infty}^{+\infty} \frac{\partial}{\partial \alpha_{k}} \frac{\partial}{\partial \alpha_{l}}|\boldsymbol{\xi}|^{\nu}\left\langle\varphi_{k}\right| \delta\left(\left(a \otimes I_{3}\right)^{\prime} \mathbf{r}-\boldsymbol{\xi}\right) \\
& \times\left.\left|\varphi_{l}\right\rangle\right|_{\alpha_{k}, \alpha_{l}=0} d \boldsymbol{\xi},
\end{aligned}
$$

where

$$
\begin{aligned}
\left\langle\varphi_{k}\right| & \delta\left(\left(a \otimes I_{3}\right)^{\prime} \mathbf{r}-\xi\right)\left|\varphi_{l}\right\rangle \\
= & \lim _{\beta \rightarrow \infty}\left(\frac{\beta}{\pi}\right)^{3 / 2}\left\langle\varphi_{k}\right| \exp \left\{-\beta \mathbf{r}^{\prime}\left(a a^{\prime} \otimes I_{3}\right) \mathbf{r}\right. \\
& \left.+2 \beta\left[\left(a \otimes I_{3}\right) \xi\right]^{\prime} \mathbf{r}-\beta \boldsymbol{\xi}^{2}\right\}\left|\varphi_{l}\right\rangle .
\end{aligned}
$$

It should be noted that integrals involving delta function (39) with some particular choice of vector $a$ can be very useful in evaluating matrix elements of many important quantities. Even though here the evaluation of potential-energy matrix elements is of concern, we will assume that $a$ is some general vector when deriving integral (41).
Applying formula (16) in Eq. (41) we obtain

$$
\begin{aligned}
\left\langle\varphi_{k}\left|\delta\left(\left(a \otimes I_{3}\right)^{\prime} \mathbf{r}-\boldsymbol{\xi}\right)\right| \varphi_{l}\right\rangle & \\
= & \pi^{3(n-1) / 2} \lim _{\beta \rightarrow \infty} \beta^{3 / 2} \mid \mathbf{A}_{k l}+\alpha_{k} \mathbf{W}_{k}+\alpha_{l} \mathbf{W}_{l} \\
& +\left.\beta\left(a a^{\prime} \otimes I_{3}\right)\right|^{-1 / 2} \exp \left\{\beta ^ { 2 } [ ( a \otimes I _ { 3 } ) \xi ] ^ { \prime } \left[\mathbf{A}_{k l}\right.\right. \\
& \left.\left.+\alpha_{k} \mathbf{W}_{k}+\alpha_{l} \mathbf{W}_{l}+\beta\left(a a^{\prime} \otimes I_{3}\right)\right]^{-1}\left[\left(a \otimes I_{3}\right) \xi\right]\right\} \\
& \times \exp \left[-\beta \xi^{2}\right] .
\end{aligned}
$$

Taking limit (42) as $\beta$ approaches infinity can be done by expanding both the determinant and the exponent into an inverse power series with respect to $\beta$. Both the determinant and the exponent term contain an inverse of a matrix. This inverse can be computed using the Woodbury matrix identity, which has the following form:

$$
(A+U B V)^{-1}=A^{-1}-A^{-1} U\left(B^{-1}+V A^{-1} U\right)^{-1} V A^{-1},
$$

where $A$ is a $p \times p$ matrix, $U$ is a $p \times q$ matrix, $V$ is a $q \times p$ matrix, and $B$ is a $q \times q$ matrix. The Woodbury identity gives an explicit form of the inverse of a rank $q$ update of some initial matrix $A$. In our case, the initial matrix [analog of $A$ in Eq. (43)] is the following $3 n \times 3 n$ matrix:

$$
\mathbf{G}_{k l}=\mathbf{A}_{k l}+\alpha_{k} \mathbf{W}_{k}+\alpha_{l} \mathbf{W}_{l} .
$$

The analog of $U B V$ in Eq. (43) is the following matrix:

$$
\beta\left(a a^{\prime} \otimes I_{3}\right)=\left(a \otimes I_{3}\right)\left(\beta I_{3}\right)\left(a^{\prime} \otimes I_{3}\right) .
$$

Applying the Woodbury identity to the inverse matrix mentioned before yields

$$
\begin{aligned}
{\left[\mathbf{G}_{k l}+\beta\left(a a^{\prime} \otimes I_{3}\right)\right]^{-1}=} & \mathbf{G}_{k l}^{-1}-\mathbf{G}_{k l}^{-1} \\
& \times\left(a \otimes I_{3}\right)\left(\Lambda_{k l}+\frac{1}{\beta} I_{3}\right)^{-1}\left(a^{\prime} \otimes I_{3}\right) \mathbf{G}_{k l}^{-1},
\end{aligned}
$$

where

$$
\Lambda_{k l}=\left(a^{\prime} \otimes I_{3}\right) \mathbf{G}_{k l}^{-1}\left(a \otimes I_{3}\right) .
$$

Now, using the inverse power-series expansion

$$
\left(\Lambda_{k l}+\frac{1}{\beta} I_{3}\right)^{-1}=\Lambda_{k l}^{-1}-\frac{1}{\beta} \Lambda_{k l}^{-2}+\frac{1}{\beta^{2}} \Lambda_{k l}^{-3}-\frac{1}{\beta^{3}} \Lambda_{k l}^{-4}+\cdots,
$$

and after some simple multiplications with rearrangement, the limit of the expression in the exponent of Eq. (42) is

$$
\begin{aligned}
\lim _{\beta \rightarrow \infty} & \beta^{2}\left[\left(a \otimes I_{3}\right) \xi\right]^{\prime}\left[\mathbf{G}_{k l}+\beta\left(a a^{\prime} \otimes I_{3}\right)\right]^{-1}\left[\left(a \otimes I_{3}\right) \xi\right]-\beta \boldsymbol{\xi}^{2} \\
& =-\boldsymbol{\xi}^{\prime} \Lambda_{k l} \boldsymbol{\xi} .
\end{aligned}
$$

Let us note that the determinant in Eq. (42) can be written as a determinant of the same inverse matrix that appears in the exponent 


$$
\begin{aligned}
\mid \mathbf{G}_{k l} & +\left.\beta\left(a a^{\prime} \otimes I_{3}\right)\right|^{-1 / 2} \\
& =\left|\left[\mathbf{G}_{k l}+\beta\left(a a^{\prime} \otimes I_{3}\right)\right]^{-1}\right|^{1 / 2} \\
& =\left|\mathbf{G}_{k l}\right|^{-1 / 2}\left|I_{3 n}-\left(a \otimes I_{3}\right)\left(\Lambda_{k l}+\frac{1}{\beta} I_{3}\right)^{-1}\left(a^{\prime} \otimes I_{3}\right) \mathbf{G}_{k l}^{-1}\right|^{1 / 2} .
\end{aligned}
$$

Here, again the expansion (48) is used and the well-known Sylvester's determinant theorem is applied, which says that if $A$ and $B$ are $p \times q$ matrices then,

$$
\left|I_{p}+A B^{\prime}\right|=\left|I_{q}+B^{\prime} A\right| .
$$

The limit of the pre-exponential factor in Eq. (42) can then be easily evaluated,

$$
\lim _{\beta \rightarrow \infty} \beta^{3 / 2}\left|\mathbf{G}_{k l}+\beta\left(a a^{\prime} \otimes I_{3}\right)\right|^{-1 / 2}=\left|\mathbf{G}_{k l}\right|^{-1 / 2}\left|\Lambda_{k l}\right|^{-1 / 2} .
$$

With this, the expression for integral (42) can be written as

$$
\begin{aligned}
\left\langle\varphi_{k}\right| & \delta\left(\left(a \otimes I_{3}\right)^{\prime} \mathbf{r}-\boldsymbol{\xi}\right)\left|\varphi_{l}\right\rangle \\
& =\pi^{3(n-1) / 2}\left|\mathbf{G}_{k l}\right|^{-1 / 2}\left|\Lambda_{k l}\right|^{-1 / 2} \exp \left[-\boldsymbol{\xi}^{\prime} \Lambda_{k l}^{-1} \boldsymbol{\xi}\right] \\
& \equiv \Upsilon_{k l} .
\end{aligned}
$$

The next step is to differentiate Eq. (53) with respect to $\alpha_{k}$ and $\alpha_{l}$ and set $\alpha_{k}=\alpha_{l}=0$. To accomplish the differentiation, let us give the explicit formula for the differential of matrix $\left(Y^{\prime} X^{-1} Y\right)^{-1}$, where $X$ is some arbitrary square matrix and $Y$ is a rectangular constant matrix,

$$
d\left(Y^{\prime} X^{-1} Y\right)^{-1}=\left(Y^{\prime} X^{-1} Y\right)^{-1} Y^{\prime} X^{-1}(d X) X^{-1} Y\left(Y^{\prime} X^{-1} Y\right)^{-1} .
$$

Also, notice that the following product, which will appear in the expression for the integral with delta function, can be simplified,

$$
\left(a^{\prime} \otimes I_{3}\right) \mathbf{A}_{k l}^{-1}\left(a \otimes I_{3}\right)=a^{\prime} A_{k l}^{-1} a \otimes I_{3}=\lambda I_{3},
$$

where $\lambda=\operatorname{tr}\left[A_{k l}^{-1} a a^{\prime}\right]$. Upon using Eqs. (54) and (55), and defining $D=a a^{\prime}, \mathbf{D}=D \otimes I_{3}$ we recover

$$
\begin{aligned}
\left.\frac{\partial}{\partial \alpha_{k}} \frac{\partial}{\partial \alpha_{l}} Y_{k l}\right|_{\alpha_{k}, \alpha_{l}=0}= & \pi^{3(n-1) / 2}\left|\mathbf{A}_{k l}\right|^{-1 / 2} \lambda^{-3 / 2} \exp \left[-\lambda \xi^{2}\right]\left\{\frac{1}{4} \eta_{1} \eta_{2}+\frac{1}{2} \eta_{3}-\frac{1}{2} \lambda^{-1}\left(\frac{1}{2} \eta_{1} \operatorname{tr}\left[\mathbf{A}_{k l}^{-1} \mathbf{W}_{l} \mathbf{A}_{k l}^{-1} \mathbf{D}\right]+\frac{1}{2} \eta_{2} \operatorname{tr}\left[\mathbf{A}_{k l}^{-1} \mathbf{W}_{k} \mathbf{A}_{k l}^{-1} \mathbf{D}\right]\right.\right. \\
& \left.+\operatorname{tr}\left[\mathbf{A}_{k l}^{-1} \mathbf{W}_{l} \mathbf{A}_{k l}^{-1} \mathbf{W}_{k} \mathbf{A}_{k l}^{-1} \mathbf{D}\right]+\operatorname{tr}\left[\mathbf{A}_{k l}^{-1} \mathbf{W}_{k} \mathbf{A}_{k l}^{-1} \mathbf{W}_{l} \mathbf{A}_{k l}^{-1} \mathbf{D}\right]\right)+\frac{1}{2} \lambda^{-2}\left(\operatorname{tr}\left[\mathbf{A}_{k l}^{-1} \mathbf{W}_{k} \mathbf{A}_{k l}^{-1} \mathbf{W}_{l} \mathbf{A}_{k l}^{-1} \mathbf{D}\right]\right. \\
& +\operatorname{tr}\left[\mathbf{A}_{k l}^{-1} \mathbf{W}_{k} \mathbf{A}_{k l}^{-1} \mathbf{D}\right] \operatorname{tr}\left[\mathbf{A}_{k l}^{-1} \mathbf{W}_{l} \mathbf{A}_{k l}^{-1} \mathbf{D}\right]+\eta_{1} \xi^{\prime}\left(a^{\prime} \otimes I_{3}\right) \mathbf{A}_{k l}^{-1} \mathbf{W}_{l} \mathbf{A}_{k l}^{-1}\left(a \otimes I_{3}\right) \boldsymbol{\xi}+\eta_{2} \xi^{\prime}\left(a^{\prime} \otimes I_{3}\right) \mathbf{A}_{k l}^{-1} \mathbf{W}_{k} \mathbf{A}_{k l}^{-1}(a \\
& \left.\left.\otimes I_{3}\right) \boldsymbol{\xi}+2 \boldsymbol{\xi}^{\prime}\left(a^{\prime} \otimes I_{3}\right) \mathbf{A}_{k l}^{-1} \mathbf{W}_{k} \mathbf{A}_{k l}^{-1} \mathbf{W}_{l} \mathbf{A}_{k l}\left(a \otimes I_{3}\right) \boldsymbol{\xi}+2 \boldsymbol{\xi}^{\prime}\left(a^{\prime} \otimes I_{3}\right) \mathbf{A}_{k l}^{-1} \mathbf{W}_{l} \mathbf{A}_{k l}^{-1} \mathbf{W}_{k} \mathbf{A}_{k l}\left(a \otimes I_{3}\right) \boldsymbol{\xi}\right) \\
& -\lambda^{-3}\left(\frac{1}{2} \operatorname{tr}\left[\mathbf{A}_{k l}^{-1} \mathbf{W}_{k} \mathbf{A}_{k l}^{-1} \mathbf{D}\right] \boldsymbol{\xi}^{\prime}\left(a^{\prime} \otimes I_{3}\right) \mathbf{A}_{k l}^{-1} \mathbf{W}_{l} \mathbf{A}_{k l}^{-1}\left(a \otimes I_{3}\right) \boldsymbol{\xi}+\frac{1}{2} \operatorname{tr}\left[\mathbf{A}_{k l}^{-1} \mathbf{W}_{l} \mathbf{A}_{k l}^{-1} \mathbf{D}\right] \boldsymbol{\xi}^{\prime}\left(a^{\prime} \otimes I_{3}\right) \mathbf{A}_{k l}^{-1} \mathbf{W}_{k} \mathbf{A}_{k l}^{-1}\right. \\
& \times\left(a \otimes I_{3}\right) \boldsymbol{\xi}+\boldsymbol{\xi}^{\prime}\left(a^{\prime} \otimes I_{3}\right) \mathbf{A}_{k l}^{-1} \mathbf{W}_{k} \mathbf{A}_{k l}^{-1}\left(a \otimes I_{3}\right)\left(a^{\prime} \otimes I_{3}\right) \mathbf{A}_{k l}^{-1} \mathbf{W}_{l} \mathbf{A}_{k l}^{-1}\left(a \otimes I_{3}\right) \boldsymbol{\xi}+\boldsymbol{\xi}^{\prime}\left(a^{\prime} \otimes I_{3}\right) \mathbf{A}_{k l}^{-1} \mathbf{W}_{l} \mathbf{A}_{k l}^{-1}\left(a \otimes I_{3}\right) \\
& \left.\times\left(a^{\prime} \otimes I_{3}\right) \mathbf{A}_{k l}^{-1} \mathbf{W}_{k} l \mathbf{A}_{k l}^{-1}\left(a \otimes I_{3}\right) \boldsymbol{\xi}\right)+\lambda^{-4}\left(\boldsymbol{\xi}^{\prime}\left(a^{\prime} \otimes I_{3}\right) \mathbf{A}_{k l}^{-1} \mathbf{W}_{k} \mathbf{A}_{k l}^{-1}\right. \\
& \left.\left.\times\left(a \otimes I_{3}\right) \xi \xi^{\prime}\left(a^{\prime} \otimes I_{3}\right) \mathbf{A}_{k l}^{-1} \mathbf{W}_{l} \mathbf{A}_{k l}^{-1}\left(a \otimes I_{3}\right) \xi\right)\right\}
\end{aligned}
$$

The above lengthy expression is the most general formula for the integral that involves delta function (39).

The final step in deriving the formula for the $\left\langle\phi_{k}\left|r_{i j}^{\nu}\right| \phi_{l}\right\rangle$ matrix element is to integrate over $\boldsymbol{\xi}=\left\{\xi_{x}, \xi_{y}, \xi_{z}\right\}$. Here we need to specify a particular form of $a$. As was mentioned above, in case of $r_{i j}$ we simply set $a=e^{i}-e^{j}$. If the matrix element that contains $r_{i}$ is needed, then we set $a=e^{i}$. In place of $a a^{\prime}$ and $a a^{\prime} \otimes I_{3}$ we will use matrices $J$ and $\mathbf{J}=J \otimes I_{3}$, respectively. Matrix $J$ has a very simple structure,

$$
J= \begin{cases}E_{i i}, & i=j \text { for } r_{i} \\ E_{i i}+E_{j j}-E_{i j}-E_{j i}, & i \neq j \text { for } r_{i j},\end{cases}
$$

where $E_{i j}$ is a matrix with 1 in the $i, j$ th position and 0 's elsewhere.
There are three types of integrals involved when Eq. (56) is integrated. These integrals have the following general forms:

$$
\begin{gathered}
\int_{-\infty}^{+\infty}|\boldsymbol{\xi}|^{\nu} \exp \left[-\lambda \boldsymbol{\xi}^{2}\right] d \boldsymbol{\xi}, \\
\int_{-\infty}^{+\infty}|\boldsymbol{\xi}|^{\nu}\left(\boldsymbol{\xi}^{\prime} \Omega \boldsymbol{\xi}\right) \exp \left[-\lambda \xi^{2}\right] d \boldsymbol{\xi}, \\
\int_{-\infty}^{+\infty}|\boldsymbol{\xi}|^{\nu}\left(\boldsymbol{\xi}^{\prime} \Pi \boldsymbol{\xi}\right)\left(\boldsymbol{\xi}^{\prime} \Theta \boldsymbol{\xi}\right) \exp \left[-\lambda \boldsymbol{\xi}^{2}\right] d \boldsymbol{\xi},
\end{gathered}
$$

where $\lambda=\operatorname{tr}\left[A_{k l}^{-1} J\right]$ and $\Omega, \Pi$, and $\Theta$ are certain $3 \times 3$ matrices. 
Integral (58) is a table integral with the following value:

$$
\int_{-\infty}^{+\infty}|\boldsymbol{\xi}|^{\nu} \exp \left[-\lambda \boldsymbol{\xi}^{2}\right] d \boldsymbol{\xi}=2 \pi \Gamma\left(\frac{3+\nu}{2}\right) \lambda^{(3+\nu) / 2} .
$$

In order to evaluate the second of the three integrals, integral (59), we can write the quadratic form $\xi^{\prime} \Omega \xi$, where $\Omega$ is a $3 \times 3$ matrix, explicitly, term by term,

$$
\begin{aligned}
\boldsymbol{\xi}^{\prime} \Omega \boldsymbol{\xi}= & \Omega_{11} \xi_{x}^{2}+\Omega_{22} \xi_{y}^{2}+\Omega_{33} \xi_{x}^{2}+2 \Omega_{12} \xi_{x} \xi_{y}+2 \Omega_{13} \xi_{x} \xi_{z} \\
& +2 \Omega_{23} \xi_{y} \xi_{z} .
\end{aligned}
$$

The diagonal terms involving $\xi_{x}^{2}, \xi_{y}^{2}$, and $\xi_{z}^{2}$ are even functions and lead to nonzero integrals. The off-diagonal terms are odd functions and the integrals involving these terms vanish. Since the integrals involving $\xi_{x}^{2}, \xi_{y}^{2}$, and $\xi_{z}^{2}$ obviously have the same value (apart from their factors, $\Omega_{11}, \Omega_{22}, \Omega_{33}$ ) we can write the resulting integral as

$$
\begin{aligned}
& \operatorname{tr}[\Omega] \int_{-\infty}^{+\infty}|\boldsymbol{\xi}|^{\nu} \boldsymbol{\xi}_{x}^{2} \exp \left[-\lambda \boldsymbol{\xi}^{2}\right] d \boldsymbol{\xi} \\
& \quad=\frac{1}{3} \operatorname{tr}[\Omega] \int_{-\infty}^{+\infty}|\boldsymbol{\xi}|^{\nu} \boldsymbol{\xi}^{2} \exp \left[-\lambda \boldsymbol{\xi}^{2}\right] d \boldsymbol{\xi} .
\end{aligned}
$$

The latter integral is a table one, similar to Eq. (61). Thus, for Eq. (59) we have

$$
\int_{-\infty}^{+\infty} \mid \xi^{\nu}\left(\xi^{\prime} \Omega \xi\right) \exp \left[-\lambda \xi^{2}\right] d \xi=\frac{2 \pi}{3} \Gamma\left(\frac{5+\nu}{2}\right) \operatorname{tr}[\Omega] \lambda^{(5+\nu) / 2} .
$$

The same procedure can be also applied to evaluate Eq. (60), where the only nonzero integrals are those involving even functions such as $\xi_{x}^{4}, \xi_{x}^{2} \xi_{y}^{2}$, etc. All other terms involving such odd functions as $\xi_{x} \xi_{y}^{3}$ or $\xi_{x} \xi_{y} \xi_{z}^{2}$ vanish. With that, one can obtain the following formula for integral (60):

$$
\begin{aligned}
& \int_{-\infty}^{+\infty}|\boldsymbol{\xi}|^{\nu}\left(\boldsymbol{\xi}^{\prime} \Pi \boldsymbol{\xi}\right)\left(\boldsymbol{\xi}^{\prime} \Theta \boldsymbol{\xi}\right) \exp \left[-\lambda \boldsymbol{\xi}^{2}\right] d \boldsymbol{\xi} \\
& \quad=\frac{2 \pi}{15} \Gamma\left(\frac{7+\nu}{2}\right) \lambda^{(7+\nu) / 2}(2 \operatorname{tr}[\Pi \Theta]+\operatorname{tr}[\Pi] \operatorname{tr}[\Theta]) .
\end{aligned}
$$

Using expressions (58)-(60) we can perform integration of Eq. (56) and after some rearrangement and simplification obtain the following final formula for the $\left\langle\phi_{k}\left|r_{i j}^{\nu}\right| \phi_{l}\right\rangle$ matrix element:

$$
\begin{aligned}
\left\langle\phi_{k}\left|r_{i j}^{\nu}\right| \phi_{l}\right\rangle= & \frac{2 \pi^{(3 n-1) / 2}}{\left|A_{k l}\right|^{3 / 2}} \Gamma\left(\frac{3+\nu}{2}\right) \lambda^{\nu / 2}\left\{\frac{1}{4} \eta_{1} \eta_{2}+\frac{1}{2} \eta_{3}\right. \\
& +\frac{\nu}{12} \lambda^{-1}\left(\eta_{1} \operatorname{tr}\left[\mathbf{A}_{k l}^{-1} \mathbf{W}_{l} \mathbf{A}_{k l}^{-1} \mathbf{J}\right]\right. \\
& +\eta_{2} \operatorname{tr}\left[\mathbf{A}_{k l}^{-1} \mathbf{W}_{k} \mathbf{A}_{k l}^{-1} \mathbf{J}\right]+2 \operatorname{tr}\left[\mathbf{A}_{k l}^{-1} \mathbf{W}_{k} \mathbf{A}_{k l}^{-1} \mathbf{W}_{l} \mathbf{A}_{k l}^{-1} \mathbf{J}\right] \\
& \left.+2 \operatorname{tr}\left[\mathbf{A}_{k l}^{-1} \mathbf{W}_{l} \mathbf{A}_{k l}^{-1} \mathbf{W}_{k} \mathbf{A}_{k l}^{-1} \mathbf{J}\right]\right) \\
& +\frac{\nu(\nu-2)}{60} \lambda^{-2}\left(2 \operatorname{tr}\left[\mathbf{A}_{k l}^{-1} \mathbf{W}_{k} \mathbf{A}_{k l}^{-1} \mathbf{J} \mathbf{A}_{k l}^{-1} \mathbf{W}_{l} \mathbf{A}_{k l}^{-1} \mathbf{J}\right]\right.
\end{aligned}
$$

$$
\left.\left.+\operatorname{tr}\left[\mathbf{A}_{k l}^{-1} \mathbf{W}_{k} \mathbf{A}_{k l}^{-1} \mathbf{J}\right] \operatorname{tr}\left[\mathbf{A}_{k l}^{-1} \mathbf{W}_{l} \mathbf{A}_{k l}^{-1} \mathbf{J}\right]\right)\right\} .
$$

The case when $\nu=-1$ represents the integrals that appear in the potential-energy matrix elements. In this case the matrix element becomes

$$
\begin{aligned}
\left\langle\phi_{k}\left|\frac{1}{r_{i j}}\right| \phi_{l}\right\rangle= & 2 \pi^{(3 n-1) / 2}\left|A_{k l}\right|^{-3 / 2} \lambda^{-1 / 2}\left\{\frac{1}{4} \eta_{1} \eta_{2}+\frac{1}{2} \eta_{3}\right. \\
& -\frac{1}{12} \lambda^{-1}\left(\eta_{1} \operatorname{tr}\left[\mathbf{A}_{k l}^{-1} \mathbf{W}_{l} \mathbf{A}_{k l}^{-1} \mathbf{J}\right]\right. \\
& +\eta_{2} \operatorname{tr}\left[\mathbf{A}_{k l}^{-1} \mathbf{W}_{k} \mathbf{A}_{k l}^{-1} \mathbf{J}\right]+2 \operatorname{tr}\left[\mathbf{A}_{k l}^{-1} \mathbf{W}_{k} \mathbf{A}_{k l}^{-1} \mathbf{W}_{l} \mathbf{A}_{k l}^{-1} \mathbf{J}\right] \\
& \left.+2 \operatorname{tr}\left[\mathbf{A}_{k l}^{-1} \mathbf{W}_{l} \mathbf{A}_{k l}^{-1} \mathbf{W}_{k} \mathbf{A}_{k l}^{-1} \mathbf{J}\right]\right) \\
& +\frac{1}{20} \lambda^{-2}\left(2 \operatorname{tr}\left[\mathbf{A}_{k l}^{-1} \mathbf{W}_{k} \mathbf{A}_{k l}^{-1} \mathbf{J} \mathbf{A}_{k l}^{-1} \mathbf{W}_{l} \mathbf{A}_{k l}^{-1} \mathbf{J}\right]\right. \\
& \left.\left.+\operatorname{tr}\left[\mathbf{A}_{k l}^{-1} \mathbf{W}_{k} \mathbf{A}_{k l}^{-1} \mathbf{J}\right] \operatorname{tr}\left[\mathbf{A}_{k l}^{-1} \mathbf{W}_{l} \mathbf{A}_{k l}^{-1} \mathbf{J}\right]\right)\right\}
\end{aligned}
$$

It is worth noticing that, when $\nu$ is zero, Eq. (66) should produce the formula for the overlap integral, and, as it can be easily verified, it does. Also, when $\nu=2 \mathrm{Eq}$. (66) reproduces formula (35) if we set $\mathbf{B}=\mathbf{J}$.

Finally, utilizing the fact that $\eta_{1}=\operatorname{tr}\left[\mathbf{A}_{k l}^{-1} \mathbf{W}_{k}\right]$ and $\eta_{2}$ $=\operatorname{tr}\left[\mathbf{A}_{k l}^{-1} \mathbf{W}_{k}\right]$ are zero for basis functions (8) and (11), the potential-energy integral can be reduced to

$$
\begin{aligned}
\left\langle\phi_{k}\left|\frac{1}{r_{i j}}\right| \phi_{l}\right\rangle= & 2 \pi^{(3 n-1) / 2}\left|A_{k l}\right|^{-3 / 2} \lambda^{-1 / 2} \\
& \times\left\{\frac{1}{2} \eta_{3}-\frac{1}{6} \lambda^{-1}\left(\operatorname{tr}\left[\mathbf{A}_{k l}^{-1} \mathbf{W}_{k} \mathbf{A}_{k l}^{-1} \mathbf{W}_{l} \mathbf{A}_{k l}^{-1} \mathbf{J}\right]\right.\right. \\
& \left.+\operatorname{tr}\left[\mathbf{A}_{k l}^{-1} \mathbf{W}_{l} \mathbf{A}_{k l}^{-1} \mathbf{W}_{k} \mathbf{A}_{k l}^{-1} \mathbf{J}\right]\right) \\
& +\frac{1}{20} \lambda^{-2}\left(2 \operatorname{tr}\left[\mathbf{A}_{k l}^{-1} \mathbf{W}_{k} \mathbf{A}_{k l}^{-1} \mathbf{J} \mathbf{A}_{k l}^{-1} \mathbf{W}_{l} \mathbf{A}_{k l}^{-1} \mathbf{J}\right]\right. \\
& \left.\left.+\operatorname{tr}\left[\mathbf{A}_{k l}^{-1} \mathbf{W}_{k} \mathbf{A}_{k l}^{-1} \mathbf{J}\right] \operatorname{tr}\left[\mathbf{A}_{k l}^{-1} \mathbf{W}_{l} \mathbf{A}_{k l}^{-1} \mathbf{J}\right]\right)\right\},
\end{aligned}
$$

and this concludes the derivation of the algorithm for the calculation of the potential-energy matrix element.

\section{NUMERICAL ILLUSTRATION}

The algorithms for calculating overlap and Hamiltonian matrix elements have been implemented in a computer program that carries out the variational calculation of the ground-state energy and the wave function. The program uses the standard procedure for solving the secular equation through simultaneous diagonalization of the overlap and Hamiltonian matrices. It also performs an optimization of the nonlinear parameters contained in the wave function using the conjugated gradient method. The algorithms for calculating the matrix elements were first implemented in the forms provided by the expressions described in the previous section. Since some of the matrices involved in these expressions are very sparse, a second "production" version of the 
TABLE I. Nonrelativistic energies of three carbon isotopes, ${ }^{12} \mathrm{C},{ }^{13} \mathrm{C}$, and ${ }^{14} \mathrm{C}$, and the carbon atom with infinite nuclear mass, ${ }^{\infty} \mathrm{C}$ as a function of the number of basis functions. All energies are in atomic units. In parenthesis we show the correction due to the finite-nuclear mass calculated as $E\left({ }^{x} \mathrm{C}\right)-E\left({ }^{\infty} \mathrm{C}\right)$.

\begin{tabular}{ccccc}
\hline \hline Basis Size & $E\left({ }^{12} \mathrm{C}\right)$ & $E\left({ }^{13} \mathrm{C}\right)$ & $E\left({ }^{14} \mathrm{C}\right)$ & $E\left({ }^{\infty} \mathrm{C}\right)$ \\
\hline 5 & $-37.12320387(0.001711599)$ & $-37.12333593(0.00157899)$ & $-37.12344870(0.00146622)$ & -37.12491492 \\
10 & $-37.48739372(0.00171599)$ & $-37.48752615(0.00158356)$ & $-37.48763925(0.00147046)$ & -37.48910971 \\
15 & $-37.59657279(0.00171719)$ & $-37.59670532(0.00158466)$ & $-37.59681849(0.00147149)$ & -37.59828998 \\
20 & $-37.66214873(0.00171470)$ & $-37.66228105(0.00158215)$ & $-37.66239404(0.00146916)$ & -37.66386320 \\
25 & $-37.69207132(0.00171434)$ & $-37.69220362(0.00158204)$ & $-37.69231661(0.00146905)$ & -37.69378566 \\
30 & $-37.71313236(0.00171502)$ & $-37.71326473(0.00158265)$ & $-37.71337776(0.00146962)$ & -37.71484738 \\
35 & $-37.72968478(0.00171382)$ & $-37.72981704(0.00158156)$ & $-37.72993000(0.00146860)$ & -37.73139860 \\
40 & $-37.74022531(0.00171473)$ & $-37.74035764(0.00158240)$ & $-37.74047066(0.00146938)$ & -37.74194004 \\
45 & $-37.75013443(0.00171611)$ & $-37.75026688(0.00158366)$ & $-37.75037998(0.00147056)$ & -37.75185054 \\
50 & $-37.75517818(0.00171660)$ & $-37.75531066(0.00158412)$ & $-37.75542380(0.00147098)$ & -37.75689478 \\
\hline \hline
\end{tabular}

code was developed where multiplications by zero were virtually eliminated, resulting in a 250 times increase in the speed of the calculations.

The first testing of the program was performed for the ${ }^{3} P$ state of the He atom with the two electrons occupying two $p$ orbitals. A minimal basis set of one $p$-orbital Gaussian was used in the calculations. After an energy matching produced by the standard Hartree-Fock calculation was obtained, the next test was performed for the ground ${ }^{3} P$ state of the carbon atom. Again, the calculations were carried out with a minimal set of Gaussians consisting of an $s$ orbital and an $s p$ shell. After successfully passing this test, the program was used in larger scale correlated calculations on the ground ${ }^{3} P$ state of the carbon atom.

In the carbon atom calculations, the major ${ }^{12} \mathrm{C}$ isotope of this system with the nuclear mass of $21868.66382 m_{e}$, where $m_{e}$ is the electron mass, was considered first. As the approach developed in this work explicitly includes the effects due to the finite-nucleus mass in the Hamiltonian, the nucleus mass has to be entered into the calculation. The ${ }^{12} \mathrm{C}$ calculations involved increasing the basis set of explicitly correlated Gaussians from a single function to a set of 50 functions. Initially this process involved adding one Gaussian at a time to the basis set and running 100 iterations of the conjugated gradient procedure that optimized the nonlinear $L_{k}$ parameters of all Gaussian functions in the basis set. After a set of twenty Gaussians was generated in this fashion the further enlargement of the basis set was done in subsets of five functions at a time. In Table I the results of the calculations are presented. Only energies obtained with basis sets with lengths increasing in increments of five functions are shown. After the calculations for ${ }^{12} \mathrm{C}$ were completed, the basis sets generated for this system were used to calculate the energies of two other carbon isotopes, ${ }^{13} \mathrm{C}$ and ${ }^{14} \mathrm{C}$. In those calculations only the linear-expansion coefficients were varied. The masses of the ${ }^{13} \mathrm{C}$ and ${ }^{14} \mathrm{C}$ nuclei used in the calculations were $23697.66779 m_{e}$ and $25520.35057 m_{e}$, respectively. The energies obtained for these isotopes are also shown in Table I along with the energies obtained for ${ }^{\infty} \mathrm{C}$, i.e., for an infinite mass of the carbon nucleus.

It is clear that with only 50 Gaussians the total energies obtained in the calculations are far from being converged. A high-quality calculation of the ground state of the carbon atom would require the use of thousands of correlated Gaussians. However, the purpose of the present calculations was to demonstrate that our algorithm produces energies consistent with the values obtained in previous carbon atom calculations. For example, our best energy value for ${ }^{\infty} \mathrm{C}$ of -37.756 89478 a.u. obtained with 50 Gaussians compares well with the one obtained by Sundholm and Olsen in the finite-element MCHF calculations of -37.792860 a.u. (the Hartree-Fock limit is -37.688619 a.u.) [20]. The next step in performing high accuracy calculations on the carbon atom and its isotopes with all-electron explicitly correlated Gaussian functions will be completed after an algorithm for calculating the energy gradient is implemented.

The present approach allows for determining the energy effect due to the finite mass of the nucleus. This effect is calculated by subtracting the ${ }^{\infty} \mathrm{C}$ energy from the energy obtained for the particular isotope. The results presented in Table I (the numbers in parentheses) the finite nuclear mass effect is, as expected, the largest for ${ }^{12} \mathrm{C}$ and the smallest for ${ }^{14} \mathrm{C}$. The results also show that the values of the mass effects are quite well converged for all isotopes within the range of the basis set sizes used in the present calculations. One can attribute the better convergence of the mass effect than that of the total energies to cancellation of errors that likely occurs in this case. To our knowledge, this is the first time the finite-nuclear mass effect was calculated for the carbon isotopes using a variational approach where this effect is explicitly included in the total variational energy.

\section{SUMMARY}

In the present work, we presented general formulas for calculating the Hamiltonian and overlap matrix elements with all-electron explicitly correlated Gaussian functions for systems with either two $p$ electrons or a single $d$ electron and an arbitrary number of $s$ electrons. The formulas have been implemented and tested for correctness. The Hamiltonian used in the present approach was obtained by separating out the center-of-mass motion from the laboratory-frame Hamiltonian and explicitly includes terms due to the finite-nucleus 
mass effects. In our calculations for the carbon atom and its isotopes, these effects have been determined.

Presently, the method developed in this work can only be applied to perform calculations with small sets of explicitly correlated Gaussians. This is because the calculations involve optimization of nonlinear parameters of Gaussians, which is a very costly procedure. As we have shown in our previous atomic calculations [8-13], this optimization can be significantly accelerated by employing the analytical energy gradient determined with respect to the Gaussian nonlinear parameters. Thus, before large-scale atomic calculations are attempted with the method developed in this work, an algorithm for calculating the gradient has to be implemented. Such is the aim of the next stage of our project.

\section{ACKNOWLEDGMENTS}

This work has been supported by the National Science Foundation. We are grateful to the University of Arizona Center of Computing and Information Technology for the use of their computer resources.
[1] M. Smith, M. Brodeur, T. Brunner, S. Ettenauer, A. Lapierre, R. Ringle, V. L. Ryjkov, F. Ames, P. Bricault, G. W. F. Drake, P. Delheij, D. Lunney, F. Sarazin, and J. Dilling, Phys. Rev. Lett. 101, 202501 (2008).

[2] Z. C. Yan, W. Nortershauser, and G. W. F. Drake, Phys. Rev. Lett. 100, 243002 (2008).

[3] G. W. F. Drake and Z. C. Yan, Adv. Quantum Chem. 53, 37 (2008).

[4] M. Puchalski and K. Pachucki, Phys. Rev. A 79, 032510 (2009).

[5] M. Puchalski and K. Pachucki, Phys. Rev. A 78, 052511 (2008).

[6] M. Puchalski and K. Pachucki, Phys. Rev. A 73, 022503 (2006).

[7] K. Pachucki and J. Komasa, J. Chem. Phys. 125, 204304 (2006).

[8] M. Stanke, J. Komasa, D. Kȩdziera, S. Bubin, and L. Adamowicz, Phys. Rev. 78, 052507 (2008).

[9] M. Stanke, J. Komasa, D. Kȩdziera, S. Bubin, and L. Adamowicz, Phys. Rev. A 77, 062509 (2008).
[10] S. Bubin and L. Adamowicz, J. Chem. Phys. 128, 114107 (2008).

[11] S. Bubin and L. Adamowicz, Phys. Rev. 79, 022501 (2009).

[12] M. Stanke, D. Kȩdziera, S. Bubin, and L. Adamowicz, J. Chem. Phys. 127, 134107 (2007).

[13] M. Stanke, D. Kȩdziera, S. Bubin, and L. Adamowicz, Phys. Rev. Lett. 99, 043001 (2007).

[14] W. L. Glab, P. T. Glynn, and F. Robicheaux, Phys. Rev. A 58, 4014 (1998).

[15] W. C. Erickson, D. McConnell, and K. R. Anantharamaiah, Astrophys. J. 454, 125 (1995).

[16] K. R. Anantharamaiah, W. C. Erickson, H. E. Payne, and N. G. Kantharia, Astrophys. J. 430, 682 (1994).

[17] N. G. Kantharia and K. R. Anantharamaiah, J. Astrophys. Astron. 22, 51 (2001).

[18] A. Bande, A. Luchow, F. Della Sala, and A. Gorling, J. Chem. Phys. 124, 114114 (2006).

[19] R. N. Zare, Angular Momentum: Understanding Spatial Aspects in Chemistry and Physics (Wiley, New York, 1988).

[20] D. Sundholm and J. Olsen, Chem. Phys. Lett. 182, 497 (1991). 